\author{
I. F. MIRABEL, and D. B. SANDERS \\ Division of Physics, Mathematics and Astronomy \\ California Institute of Technology \\ Pasadena, CA 91125
}

\begin{abstract}
The majority of infrared active galaxies $\left(f_{\text {fir }} / f_{b} \geq 2\right)$ have molecular to atomic mass fractions in the range of 0.5 to 2.0. Among the galaxies with the higher infrared excesses there are spectacular cases of $\mathrm{HI}$ deficient systems, where less than $15 \%$ of the total mass of interstellar gas is in atomic form. The optical morphology of luminous infrared galaxies suggest that the overall mass fractions of molecular to atomic gas, and the infrared luminosities per nucleon of interstellar gas are enhanced during galaxy-galaxy interactions.
\end{abstract}

\title{
1. Results
}

The relations between the masses of molecular and atomic gas with the far-infrared and blue luminosities were studied in an unbiased sample of galaxies with far-infrared luminosities in the range of $2 \times 10^{10} L_{\odot}-2 \times 10^{12} L_{\odot}$. The galaxies were selected from the IRAS Bright Galaxy Sample of Soifer et al. (1987). The atomic and molecular components were determined from two large and homogeneous surveys of that sample: one in HI conducted at Arecibo by Mirabel and Sanders (1988), the other conducted in $\mathrm{CO}(1-0)$ by Sanders et al. (in progress) with the 12-m NRAO and 14-m FCRAO antennas. All the galaxies considered for the present study are unresoved by the telescopes used for the observations.

Figure 1a shows that the majority of the galaxies with infrared excesses in the range $2 \leq$ $\mathrm{f}_{\mathrm{fir}} / \mathrm{f}_{\mathrm{b}} \leq 40$ have $\mathrm{M}\left(\mathrm{H}_{2}\right) / \mathrm{M}(\mathrm{HI})$ mass ratios between 0.5 and 2 . However, among galaxies with $\mathrm{f}_{\mathrm{fir}} / \mathrm{f}_{\mathrm{b}}$ $\geq 50$ there are striking $\mathrm{HI}$ deficient systems, with less than $15 \%$ of the gas in atomic form. Despite the large scatter, Figure 1a shows an enhancement of the overall fraction of gas in molecular form with increasing far-infrared excess.

Figure lb shows that there is an enhancement of the infrared luminosity per nucleon of interstellar gas with increasing far infrared excess. Galaxies with the highest infrared excess may have infrared luminosities per nucleon that are as high as $\sim 20$ times that found for the Galaxy.

Figure 2 summarizes the morphologies of the sample galaxies as revealed by optical images obtained with the $1.5-\mathrm{m}$ and $5-\mathrm{m}$ Palomar telescopes. A striking result is that most, if not all infrared luminous galaxies appear to be interacting systems. Furthermore, advanced mergers (M) and strongly damaged disks (D) predominate among galaxies with the highest far-infrared excesses, while less strongly interacting systems $(\mathrm{C}$ and $\mathrm{P})$ predominate among galaxies with more moderate far-infrared excess. This suggests that the far-infrared excess in these galaxies is related to the intensity of the tidal interactions.

\section{References}

Mirabel, I. F., and Sanders, D. B. 1988, Ap. J., 335, in press.

Soifer, B. T., Sanders, D. B., Madore, B. F., Neugebauer, G., Persson, C. J., Persson, S. E., and Rice, W. L. 1987, Ap. J., 320, 238. 
604
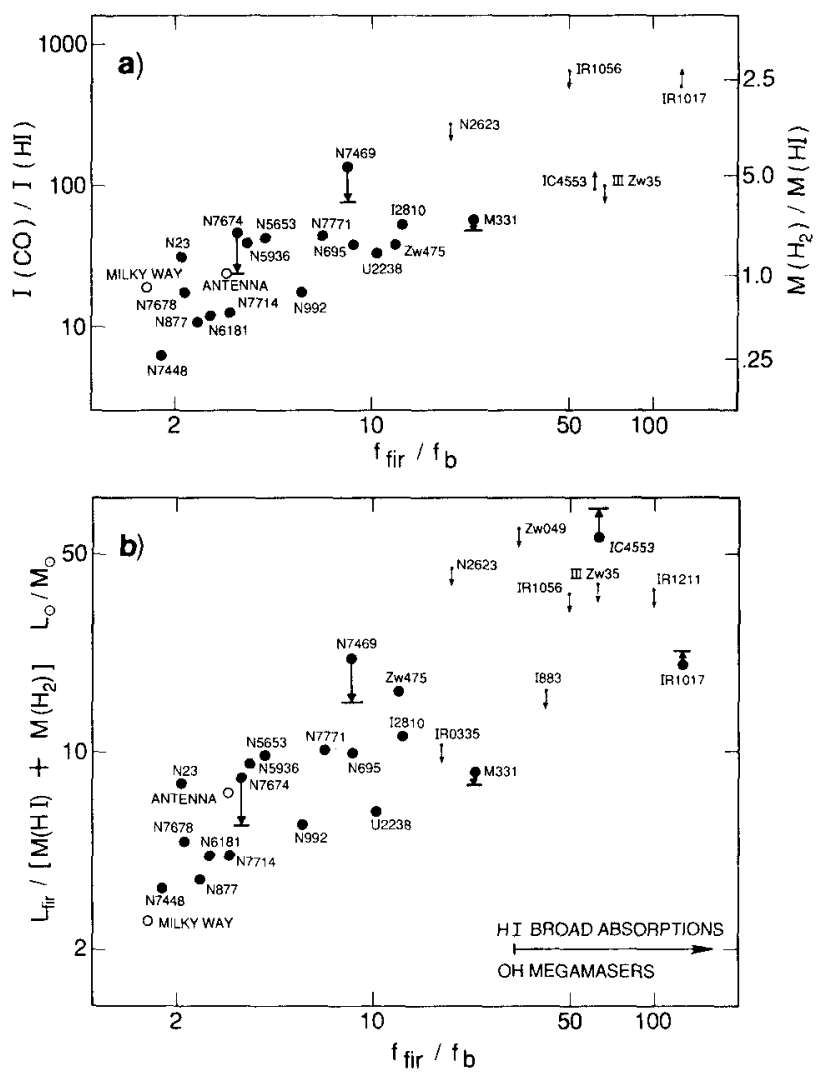

Figure 1. a) $C O(1 \rightarrow 0) / H I$ flux ratios measured at $\lambda 2.6 \mathrm{~mm}$ and $\lambda 21 \mathrm{~cm}$, as a function of the far-infrared-to-blue flux ratio, $\mathrm{f}_{\mathrm{fir}} / \mathrm{f}_{\mathrm{b}}$, for 28 luminous infrared galaxies. The arrows are due to lower and upper limits in the measurements of the HI emission. The verticle scale on the right was computed using the relation $M\left(\mathrm{H}_{2}\right)=5.82 \mathrm{~L} \mathrm{co}$. b) The far-infrared luminosity per unit mass of $H I+\mathrm{H}_{2}$ vs. $\mathrm{f}_{\mathrm{fir}} / \mathrm{f}_{\mathrm{b}}$. Most of the galaxies with $\mathrm{f}_{\mathrm{fir}} / \mathrm{f}_{\mathrm{b}} \geq 40$ exhibit HI absorption and $\mathrm{OH}$ maser emission with velocity widths of several hundred $\mathrm{km} \mathrm{s} \mathrm{s}^{-1}$.

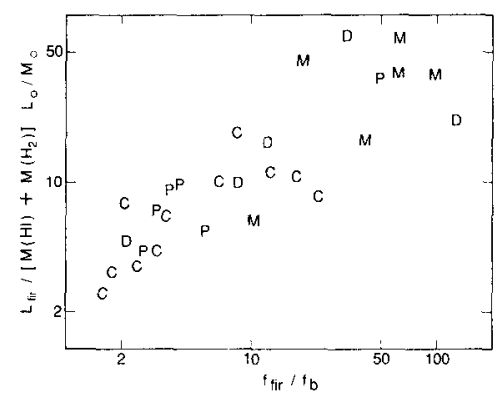

Figure 2. Morphology of the galaxies in Figure $1 b . C=$ companion $0.5-2.0$ diameters away, $P$ = companion $\leq 0.5$ diameter, $D=$ single tidally distorted disk, $M=$ advanced merger . 